\title{
DUGMO: tool for the detection of unknown genetically modified organisms with high- throughput sequencing data for pure bacterial samples
}

Julie Hurel ${ }^{1}$, Sophie Schbath ${ }^{2}$, Stéphanie Bougeard ${ }^{3}$, Mathieu Rolland ${ }^{4}$, Mauro Petrillo ${ }^{5}$ and Fabrice Touzain ${ }^{1 *}$ (D)

\author{
* Correspondence: fabrice.touzain@ \\ anses.fr \\ ${ }^{1}$ ANSES, Laboratoire de Ploufragan, \\ GVB unit, 22440 Ploufragan, France \\ Full list of author information is \\ available at the end of the article
}

\begin{abstract}
Background: The European Community has adopted very restrictive policies regarding the dissemination and use of genetically modified organisms (GMOs). In fact, a maximum threshold of $0.9 \%$ of contaminating GMOs is tolerated for a "GMOfree" label. In recent years, imports of undescribed GMOs have been detected. Their sequences are not described and therefore not detectable by conventional approaches, such as PCR.

Results: We developed DUGMO, a bioinformatics pipeline for the detection of genetically modified (GM) bacteria, including unknown GM bacteria, based on Illumina paired-end sequencing data. The method is currently focused on the detection of GM bacteria with - possibly partial - transgenes in pure bacterial samples. In the preliminary steps, coding sequences (CDSs) are aligned through two successive BLASTN against the host pangenome with relevant tuned parameters to discriminate CDSs belonging to the wild type genome (wgCDS) from potential GM coding sequences (pgmCDSs). Then, Bray-Curtis distances are calculated between the wgCDS and each pgmCDS, based on the difference of genomic vocabulary. Finally, two machine learning methods, namely the Random Forest and Generalized Linear Model, are carried out to target true GM CDS(s), based on six variables including Bray-Curtis distances and GC content. Tests carried out on a GM Bacillus subtilis showed 25 positive CDSs corresponding to the chloramphenicol resistance gene and CDSs of the inserted plasmids. On a wild type B. subtilis, no false positive sequences were detected.
\end{abstract}

Conclusion: DUGMO detects exogenous CDS, truncated, fused or highly mutated wild CDSs in high-throughput sequencing data, and was shown to be efficient at detecting GM sequences, but it might also be employed for the identification of recent horizontal gene transfers.

Keywords: Detection, Unknown GMO, Bacteria, Illumina sequencing data, Machine learning

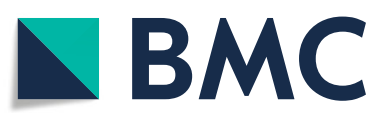

c The Author(s). 2020 Open Access This article is licensed under a Creative Commons Attribution 4.0 International License, which permits use, sharing, adaptation, distribution and reproduction in any medium or format, as long as you give appropriate credit to the original author(s) and the source, provide a link to the Creative Commons licence, and indicate if changes were made. The images or other third party material in this article are included in the article's Creative Commons licence, unless indicated otherwise in a credit line to the material. If material is not included in the article's Creative Commons licence and your intended use is not permitted by statutory regulation or exceeds the permitted use, you will need to obtain permission directly from the copyright holder. To view a copy of this licence, visit http://creativecommons.org/licenses/by/4.0/. The Creative Commons Public Domain Dedication waiver (http://creativecommons.org/publicdomain/zero/1.0/) applies to the data made available in this article, unless otherwise stated in a credit line to the data. 


\section{Background}

A genetically modified organism (GMO) is a living being "in which the genetic material has been altered in a way that does not occur naturally by mating and/or natural recombination" (European Commission, 2001) [1]. We call the host genome the wild, original genome to which a genetic modification was made to build a GMO. In this article, we focus on GMOs resulting from the integration of exogenous genes into the host genome. European legislation requires the presence of GMOs in food products to be reported when they represent more than $0.9 \%$ of the ingredients [2]. This regulation requires that all GMOs or products sourced from GMOs must be declared to the European authorities, the modifications of their nucleic acid sequences have to be recorded in appropriate databases, and a detection method based on standard molecular techniques (e.g., PCR) must be provided. The GMOMETHODS [3], Eugenius [4], and GMO-Amplicon [5] databases list the GMOs authorized on the European market and their associated detection methods. In addition, Morisset et al. 2014 [6] developed a software program called GMOseek to optimise routine GMO testing in the laboratory, thus helping decision-making at all phases of analysis. More recently, with the development of high-throughput whole genome sequencing (WGS) [1, 7-10], bioinformatics analyses of the sequencing datasets based on alignment similarities $[1,7,8]$ have been developed to characterize referenced GMO elements.

Willems et al. 2016 [11] proposed a statistical framework based on next generation sequencing (NGS) to predict the number of reads required to detect insert sequences, and thus confirm their integration into the host genome. All these tools facilitate detection work in routine laboratory testing, as long as the desired insert sequence is known. However, undescribed GMOs are also produced. For these, the sequence inserted into the host genome is completely unknown (not provided by GMO designers). Therefore, they are difficult to detect by the previously described methods. We must distinguish 3 types of unknown GM, those with only suppressed genes, those made by punctual mutations with CRISPR/TALEN technics, and finally those consisting of exogenous CDS insertion, CDS truncation or fusion with exogenous CDS. To detect this third, and most frequent, category of unknown GM, we propose a new bioinformatics tool called DUGMO, currently tailored to analyses of bacterial genomes.

Each species has its own vocabulary that can support statistical analyses. The most studied vocabulary property is codon usage [12], but longer words are used in statistical methods to decipher motifs with biological functions [13], to detect horizontal gene transfer [14], or to identify species-specific viral sequences [15] for instance. No application using these types of properties has been used in the context of GMO detection to date. DUGMO fills this gap.

DUGMO machine learning steps use two sets of CDSs. The first set corresponds to CDSs of the host genome with an extremely precise vocabulary definition. The second set corresponds to known GMO CDSs with a very wide range of vocabularies, only sketching possible diversity. To detect GM insert, DUGMO will report as GM all CDSs of the prediction set whose vocabulary differs from the one of the host genome.

DUGMO detects sequences of unknown exogenous inserts of a genetically modified (GM) bacterium. It uses high-throughput sequencing data that are previously cleaned and sorted thanks to a dedicated pipeline and two BLASTN alignments on the host pangenome (section 2.2). After the cleaning and sorting steps, a databank of known 
GMO inserts is cleaned to remove wild CDSs of the species (section 2.2). The proposed tool is based on statistical calculation of distances (section 2.3) associated with an automated learning step (section 2.4). GMOs resulting from small modifications by deletion or substitution will not be taken into account. GMOs resulting from methodologies such as CRISPR-CAS9 [16] can only be detected if they incorporate at least one exogenous CDS or involve gene truncation or fusion. Both chromosomal integration and plasmid insertion are detected.

\section{Results}

Datasets

A GM strain of B. subtilis has been sequenced at the Bavarian Health and Food Safety Authority (LGL) in Germany in the Hi-seq 1500 system [17, 18]. This dataset is called 'Data1' in the following. The paired Hi-seq 2500 sequencing data of the wild type B. subtilis 9407 strain used are available on the NCBI Sequence Read Archive (SRA) under accession number SRR8935610. This dataset is called 'Data2' in the following. The paired Mi-seq sequencing data of the three GM strains of Escherichia coli [19] used are available on the NCBI SRA under accession numbers SRR9304542, SRR9304539, and SRR9304540. These three genomes come from the same strain but each one has a distinct plasmid containing the parE gene from one of three different species: Agrobacterium tumefaciens, Mycobacterium tuberculosis and Streptococcus pyogenes. At the time of writing this article, the NGS datasets used are the only ones publicly available clearly described as GM bacteria. Two reference genomes, B. subtilis str. 168 (NC_000964.3) and E. coli str. K-12 substr. MG1655 (U00096.3) were also used. Two pangenomes were constructed, one for $B$. subtilis composed of 37 strains, and one for E. coli composed of 45 strains. Pangenomes were constructed manually to encompass the full range of analysed species and are available in the "testdata" directory of the DUGMO git repository. For B. subtilis, we took a known pangenome [20] and enriched it with wild strains from various environments available on the NCBI (list of accession numbers given in the Additional file 1, section 2). For E. coli, the 5 main species subgroups [21] were considered and enriched with newer complete PacBio genomes and plasmids from various environments available on the NCBI (list of accession numbers given in the Additional file 1, section 3). A databank of GMO coding sequences (CDSs) provided by the JRC and supplemented by insert CDSs of bacterial GMOs from the literature was created (list specified in Additional file 2, related literature references given in Additional file 3). This databank currently does not include GM CDSs of bacteria analysed in this paper. DUGMO requires paired Illumina sequencing data of a single bacterial species; it does not deal with metagenomic or multigenomic data. The JRC sequences included in the GM databank provided in this paper are obtained by PCR simulation screening of public nucleotide sequence databanks including patents and available whole plant genomes [22].

\section{Cleaning pipeline for raw data, host pangenome and known GMO databank}

The objective of this section is to describe the production of three sets of data: host genome CDSs, potential GM CDSs, and known GMO CDSs. To create these datasets, a 
pipeline was implemented to clean the raw data of the suspected GM bacterium by eliminating all sequences belonging to the reference genome. This step is frequently used in the processing of high-throughput sequencing data (e.g., refer to Baron et al. 2018 [23] to remove chromosomal reads to assemble E. coli plasmids). This pipeline sorts CDSs into two categories: CDSs of potential GMO inserts and CDSs belonging to the wild type genome. Several steps are required (Fig. 1). First, the raw sequencing data of the potential GM bacterium are assembled twice with Shovill 1.0.4 [24] with the trim option, once on the whole genome, and once focusing on plasmids (plasmid option of SPAdes 3.13.1 [25]). Scaffolds with a coverage depth of less than 3 are eliminated; this threshold is intended to remove potential contamination of the bacterial genome, such as contamination by another sequenced library or by the matrix in which the bacteria are located. These two results of assembly are annotated with Prokka 1.12 [26] with the usegenus option to predict CDSs. These CDSs are merged and filtered out for duplicated sequences. In parallel, the raw sequencing data of the potential GM bacterium are "trimmed" using Trimmomatic software 0.39 [27], and aligned by Bowtie2 2.3.4.3 [28] with the reference genome. Then, the non-aligned reads (including any GMO inserts) are aligned with the CDSs predicted in the previous Prokka CDSs using BWA MEM 0.7.17 [29]. A consensus of the covered CDSs is deduced from the BWA aligned reads using Samtools 1.9 [30]. At the end of these steps, the CDSs present in this BWA alignment consensus and containing the potential GM insert sequences are retained as potential GM CDSs. The remaining CDSs are kept as CDSs related to the host genome.

A pangenome groups together the full range of genes present in the same species. Two BLASTN 2.6.0 [31] alignments of the potential GM CDSs generated by the cleaning pipeline are performed on the pangenome of the potentially GM bacterium, one on CDSs and one on whole genomes (Additional file 1, Fig. S1). The aim of these BLASTN alignments is to carry out secondary sorting (the first was done by the cleaning pipeline) in order to separate the CDSs related to the host genome from potential GM CDSs. The first BLASTN on pangenome CDSs removes CDSs belonging to the host genome that are present in potential GM inserts (for parameters see Additional file 1, section 4.1). The second BLASTN on the pangenome aims to remove CDSs predicted by Prokka, but corresponding in our pangenome to non-coding sequences (for parameters see Additional file 1, section 4.2). The CDSs aligned at least in one of the two

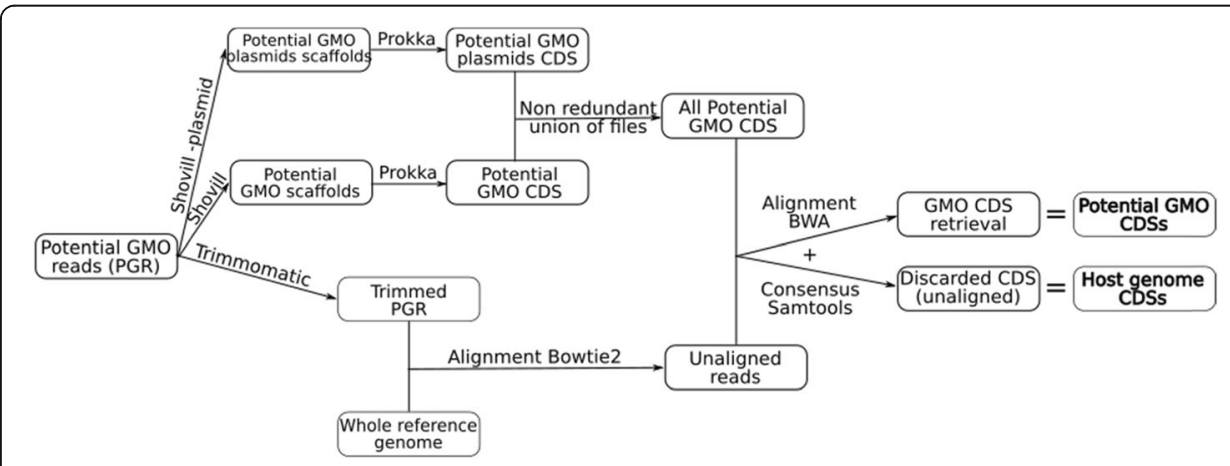

Fig. 1 Diagram of cleaning pipeline for raw data allowing preliminary sorting of the CDSs of the potential GM genome 
BLASTN steps are added to the CDSs related to the host genome defined at the end of the cleaning pipeline. The non-aligned CDSs are kept as potential GM CDSs.

After these two sorting steps, the databank of known GMO inserts is filtered to define a set of known GM CDSs used for distance calculation (section 2.3). A CDS is qualified as GM only when considering it in a setting different from the genome in which it is naturally present. When the aim is to identify whether a bacterium or an organism is a GMO or not, all CDSs naturally present in the genome of the species of the suspected strain are eliminated from our databank of GMO CDSs using a BLASTN alignment (for parameters see Additional file 1, section 4.3). In addition, the parts of CDSs aligned during this BLASTN are also removed from the CDSs in the databank, leading to a filtered bank of GMO CDSs. We computed median for each of the three distances (P L3M1, P L4M2 and F L9M7 in section 2.3) of each host genome CDS and the set of all host genome CDSs and called them respectively medL3M1, medL4M2 and medL9M7. Finally, CDSs of known GMO databank whose distances to the set of all host genome CDSs verify (P L3M1 < medL3M1 and P L4M2 < medL4M2 and F L9M7 < medL9M7) are discarded.

After the two sorting steps and filtering of the databank, we then produced three disjoint sets of CDSs in which each CDS is individually compared to the set of all host genome CDSs: (a) the filtered bank of GMO CDSs, (b) the host genome CDSs, and (c) the potential GM CDSs.

\section{Vocabulary of the host genome and bray-Curtis (BC) distances General information}

To assess whether or not each potentially GM CDS is distant from the CDSs of the host genome, a distance calculation is proposed with the idea that the greater the distance, the higher the probability that a CDS will be part of a GM insert. Our hypothesis is that the insert CDS introduced into the unknown GMO should have a different "vocabulary" from that of the genome at the source of the GMO. In fact, a genome has its own vocabulary, made up of words. A word is a short sequence of nucleotides with a predefined length, such as "ATGCCT". The vocabulary, denoted by $M$, that is used in our method can be either the set of all words of a given length $l$ or a subset of these words, specific to the host genome. In the second case, the word selection is made initially by using R'MES software [32] that searches for exceptional words in a given sequence, i.e. significantly over- or under-represented words with respect to a given Markov model. For each word of a given length, R'MES computes a score of exceptionality that measures the significance of the difference between its observed and expected number of occurrences. For this, the distribution of the number of occurrences is approximated by Gaussian distribution, well adapted for frequent words.

\section{Bray-Curtis $(B C)$ distances}

After comparing several alternative distances (Additional file 1, section 5), the most relevant Bray-Curtis dissimilarity was selected. This dissimilarity index does not verify the triangle inequality so it should not be called a distance. However, to facilitate understanding, the term "Bray-Curtis distance" will be used in this article. The BrayCurtis distance is initially an ecological distance [33] to evaluate the dissimilarity 
between two given samples in terms of the abundance of available species. This distance is equal to 1 when the two compared datasets have no common point, and 0 when they have identical compositions. Our aim is to compare a sequence $S$ and a set of sequences $H$ based on their word compositions, via the following equation

$$
B C(S, H)=1-\frac{2 \sum_{m \in M}[f(m, S)+f(m, H)]}{\sum_{m \in M}[f(m, S)+f(m, H)]}
$$

where $M$ is the set of the selected words of length $l$ (in case of no selection, $M$ is the set of all the $4^{l}$ possible words of length $l$ in the alphabet $\left.\{A, C, G, T\}\right), m$ is one word in $M, f$ is a function set either to $F$ (for frequencies; defined in the following Eq. (2)) or $P$ (for proportions; defined in the following Eq. (3)), and relates to the count of motif $m$ in the ad-hoc sequence. In practice, $S$ is typically a CDS (potential GM or not) and $H$ is the set of deduced host CDSs, leading to a notable difference in the cumulated length of these two sequence sets, and then in the order of magnitude of the counts in $S$ and $H$. To circumvent this problem, the Bray-Curtis distance is computed on normalized counts, and two normalizations are considered, called in frequencies versus in proportions, as explained below.

\section{$B C$ distance in frequencies}

The $f$ function in Eq. (1) is set to

$$
f(m, S)=F(m, S):=\frac{C(m, S)}{\sum_{w \in M} C(w, S)}
$$

where $C(m, S)$ denotes the number of occurrences - or count - of word $m$ in sequence $S$, and $w$ is every word in $M$. In other words, $\mathrm{f}(\mathrm{m}, \mathrm{S})$ is the normalized frequency of $\mathrm{m}$ in S. For this calculation of Bray-Curtis distance, only the nucleotides at the third position of the codons of the CDSs used are considered, denoted by $\mathrm{CDS}_{3}$. The justification for the concatenation of the third position of the codons is given in the Additional file 1 , section 6 . The $\mathrm{CDS}_{3}$ set allows us to consider words of size $n$ containing the useful information of words of size $3 n$ in the entire CDSs.

\section{$B C$ distance in proportions}

The $f$ function in Eq. (1) is set to

$$
f(m, S)=P_{H}(m, S):=C(m, S) \times \frac{\sum_{w \in M} C(w, H)}{\sum_{w \in M} C(w, S)} .
$$

In other words, $f(m, S)$ is a normalization of the count of $m$ in $S$ equivalent to consider $m$ in a sequence as large as the cumulated length of $H$. Note that with this definition, when $S=H$, we get $f(m, H)=C(m, H)$.

\section{Preliminary results}

In an initial step, the $\mathrm{BC}$ distances are computed both in frequencies and in proportions, with words of length $l$ from 3 to 9 , maximum Markov models of order $k=l-2$ and different ratios of selected words. These combinations of parameters are tested on our datasets Data1 and Data2, in which the GM bacterium status is known (section 2.1) to better separate the host CDSs from the GM CDSs in terms of distance. Three 
different combinations are chosen to be used jointly in the machine learning step described below:

(P L3M1): BC in proportions for all 3-letter words under Markov model of order 1.

(P L4M2): BC in proportions for all 4-letter words under Markov model of order 2.

(F L9M7): BC in frequencies for the $10 \%$ most over-represented 9-letter words under Markov model of order 7.

P L3M1 was chosen because this calculation characterizes the three-letter words of the CDSs, which is specific to the host genome vocabulary, including codon usage. F L9M7 uses long over-represented words (due to the concatenation of the third position of the codon) to make the words found in the compared CDS very specific (because of length) and fitted to the host genome codon usage (considering only third letters of CDS 27-letter words). P L4M2 allows us to characterize CDS by using small and therefore more frequent words distinct from codon usage, in order to obtain a more accurate distribution of words on the CDS.

These Bray-Curtis distance computations (P L3M1, P L4M2 and F L9M7) are performed on each of the three sets of CDSs obtained at the end of the cleaning pipeline (section 2.2), as summarized in Fig. 2, in order to prepare the machine learning step (next paragraph). Note that only CDSs with a length greater than or equal to 27 nucleotides are used, because of the minimum length of the word size considered in the calculation of the Bray-Curtis distance in frequencies.

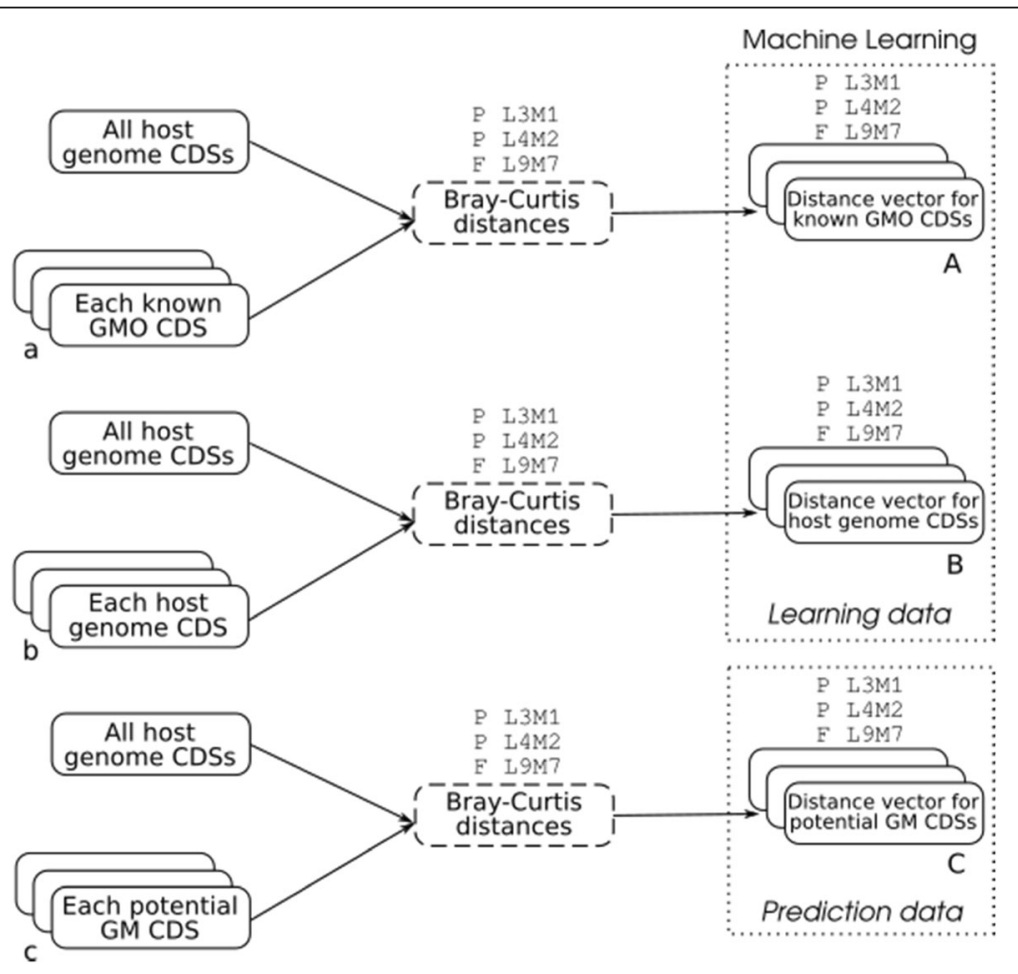

Fig. 2 Bray-Curtis distances in proportions (L3M1, L4M2) and frequencies (L9M7) applied to obtain learning and prediction data used in the machine learning step 
Prediction of GM inserts with a machine learning procedure and selection of the method Methods

Our aim is to target true GM insert CDSs. To this end, a large number of classification methods were applied, within a machine learning procedure (caret 6.0_81 package in $\mathrm{R}$ 3.5.1 [34]), and the most efficient method in terms of predicting GM CDSs was selected. The 12 classification methods used were: 4 parametric methods: Generalized Linear Model (logit), Stepwise Linear Discriminant Analysis (stepLDA), Stepwise Quadratic Discriminant Analysis (stepQDA) and Partial Least Squares (plsda); 8 nonparametric methods: Neural Networks (nnet), Support Vector Machines with Radial Basis Function Kernel (svmRadial), K-Nearest Neighbours (knn), as well as 5 based on decision trees: Classification Tree (Rpart), Random Forest (RF), Bagging Classification (Treebag), Extreme Gradient Boosting Tree (xgboost), and the supervised classification algorithm C5.0. In this process, a K-fold cross-validation procedure was used. It consists in splitting the whole dataset into $\mathrm{K}$ subsets, namely a calibration set (consisting of $\mathrm{K}-1$ subsets) and a validation set (the $\mathrm{K}$ remaining subset). In a first step, the calibration set is used to select the parameters of the model and its fitting ability. In a second step, this model is validated on the prediction set to get its prediction ability. This calibration and prediction processes were repeated $\mathrm{K}$ times. The parameters of each machine learning method were optimised following a 10-fold cross-validation procedure. The performances of these methods were compared on the basis of a 2-fold crossvalidation, in which data were previously stratified and centred. It is worth noting that stratified sampling provides homogeneous distribution of GM CDSs in the sampled data.

\section{Criteria to compare methods}

B. subtilis GM (Data1) was used to select the best predictive method, four performance criteria were proposed: false negative rate, specificity, sensitivity [35], and false positive rate. Among these four criteria, the most crucial one was that the method should not predict a GM insert CDS as a host genome CDS (i.e., no false negatives). The importance of the variables of each method were also calculated. For each method, the ROC curve and the importance of each variable usage among the six available variables were calculated (Additional file 1, section 7).

\section{Data}

The learning data were the CDSs of the host genome and the databank of filtered known GMO CDSs (Fig. 2a and b). CDSs encoding RNAs were deleted from these learning data. The prediction data were the candidate GM CDSs (Fig. 2c) that did not align with the pangenome (Section 2.2). The associated variables were the Bray-Curtis distances in proportions L4M2, L3M1 and in frequencies L9M7, the length of the CDS, the average $A_{H}(S)$ of the exceptionality scores provided by R'MES in the host genome for L4M2 and L9M7 (see Eq. 4), the count density per nucleotide in the CDS for 4 and 9-letter words (sum of the counts $C(m, S)$ of all selected words $m$, divided by the length of the CDS $S$ ) and the CDS GC content. The count density per nucleotide is a way of measuring the proportion of the host exceptional words in the CDS. The average of 
exceptionality scores is a measure of exceptionality of the words found in the CDS, considering host CDSs composition

$$
A_{H}(S)=\frac{\sum_{m \in M} K(m, H) \times C(m, S)}{\sum_{m \in M} C(m, S)}
$$

where $K(m, H)$ is the R'MES exceptionality score of one word $m$ in the host CDSs $H$.

\section{Results}

The results, illustrated in Fig. 3, were obtained from the 12 classification methods run 50 times and applied to the learning data (Fig. 2a and b) of the B. subtilis GM bacteria (Data1). These process redundancies ensured robustness of results, some methods being non-deterministic.

The RF, C5.0, Treebag, and xgboost methods gave the best results on calibration and prediction. For calibration purposes, the RF, Treebag and C5.0 methods had a sensitivity and specificity of 1 , a false positive and a false negative rate of 0 . The xgboost method obtained 0.99 in sensitivity and specificity, and a false positive and a false negative rate of 0.01 . For prediction purposes, RF and C5.0 had a sensitivity and a specificity of 0.98 , with a positive and a false negative rate of 0.01 . The Treebag and xgboost methods had a sensitivity and a specificity of 0.97 with a false positive and a false negative rate of 0.015 . For the logit method, the prediction data and the calibration data had the same results. The logit method had a sensitivity of 0.95 and a specificity of 0.94 , with a false positive rate of 0.05 and a false negative rate of 0.04 .

Following our performance criteria (i.e., false negative rate, specificity, sensitivity and false positive rate), the non-redundant union of positive results of RF and logit methods was retained for the automatic learning step. Then, global sensitivity and specificity of the program were deduced (Additional file 1, section 7.1). The most important variables for predicting CDSs with the Random Forest method were the GC content and the Bray-Curtis distance in proportions L4M2, and with the logit method, Bray-Curtis distances in frequencies L9M7 and proportions L3M1 (Additional file 1, section 7.2). They have complementary selection criteria according to their variable importance, and do not belong to the same method family.

\section{Application of DUGMO on real data}

DUGMO was developed using the data from B. subtilis (i.e., Data1, Data2). Validation was conducted on E. coli genomes. Table 1 summarizes DUGMO results on the two B. subtilis genomes (i.e., Data1, Data2) and on the three E. coli genomes. The GM CDSs on GM B. subtilis (Data1), correctly predicted by DUGMO, are the main genes located on the plasmids (riboflavin) and the gene conferring resistance to chloramphenicol. Combining the results of the RF and logit methods provides added value for accurately identifying potential GM inserts. At the end of the automatic learning step for the wild type B. subtilis (Data2), no CDS was predicted as a GM insert (see line GM B. subtilis and Machine learning column in Table 1). With the GM E. coli carrying genes of A. tumefaciens, $M$. tuberculosis or S. pyogenes, the results contained respectively 5, 4 and 5 CDSs predicted as GM inserts. However, one false positive was found in the results of E. coli carrying genes of S. pyogenes. The CDS found corresponds to an annotated aslA gene present in E. coli strongly truncated in our strain (984 nt instead of 


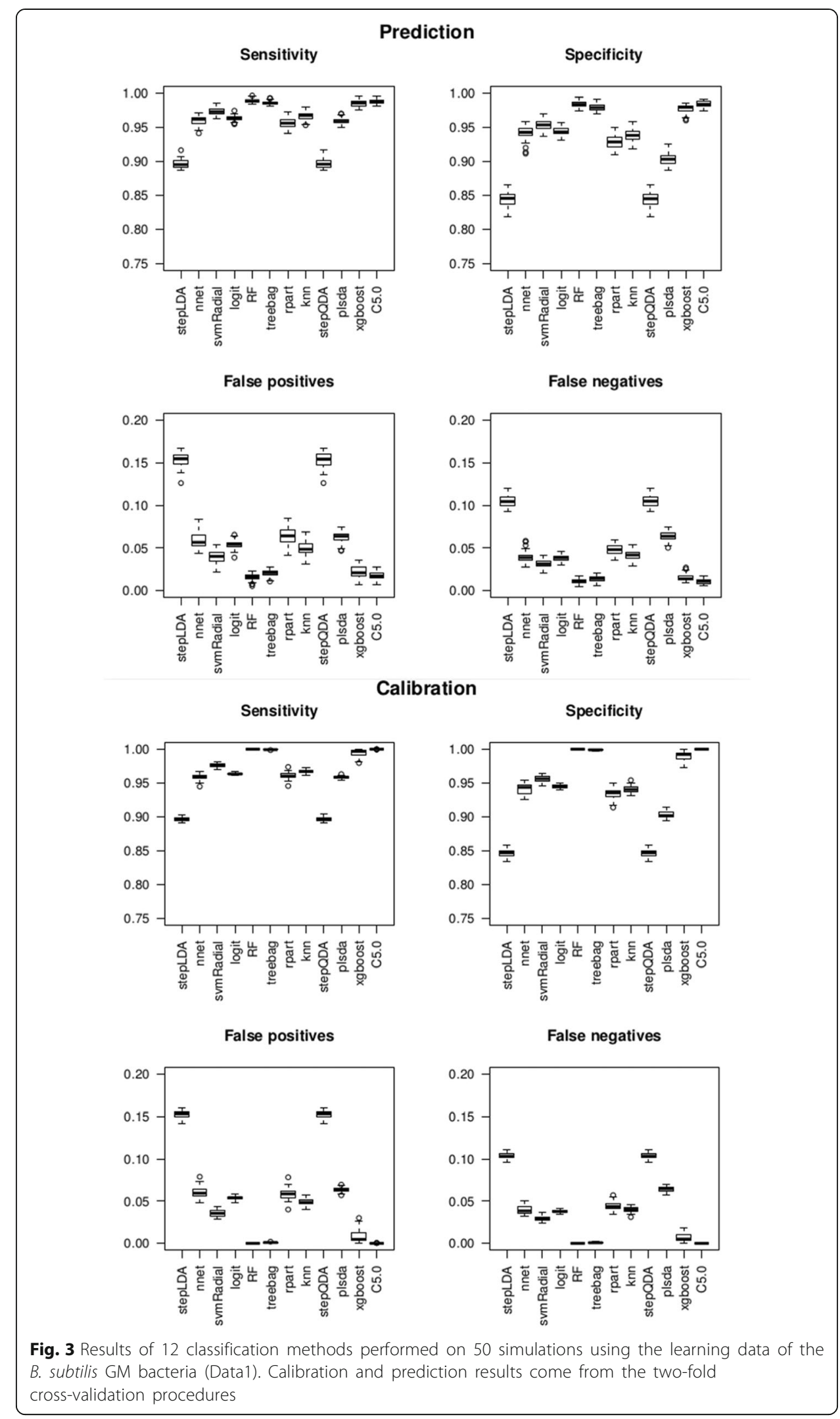


Table 1 DUGMO results for two B. subtilis genomes (i.e., Data1, Data2) and three E. coli genomes

\begin{tabular}{|c|c|c|c|c|c|c|c|}
\hline & \multicolumn{3}{|c|}{ Machine Learning } & & & & \\
\hline & \multicolumn{2}{|l|}{ Learning data } & \multirow{2}{*}{$\begin{array}{l}\text { Prediction } \\
\text { data } \\
\text { Number of } \\
\text { potential GM } \\
\text { CDSs (1) }\end{array}$} & \multicolumn{4}{|c|}{ DUGMO final results } \\
\hline & $\begin{array}{l}\text { Number of } \\
\text { host genome } \\
\text { CDSs (1) }\end{array}$ & $\begin{array}{l}\text { Number of } \\
\text { known GMO } \\
\text { CDSs (2) }\end{array}$ & & $\begin{array}{l}\text { True } \\
\text { positives } \\
\text { (3) }\end{array}$ & $\begin{array}{l}\text { False } \\
\text { positives } \\
\text { (3) }\end{array}$ & $\begin{array}{l}\text { Max false } \\
\text { negatives } \\
\text { (3) (4) }\end{array}$ & $\begin{array}{l}\text { True } \\
\text { negatives } \\
\text { (3) }\end{array}$ \\
\hline $\begin{array}{l}\text { GM B. subtilis } \\
\text { (Data1) }\end{array}$ & 4102 & 2714 & 39 & 25 & 0 & 12 & 2 \\
\hline $\begin{array}{l}\text { Wild type B. subtilis } \\
\text { (Data2) }\end{array}$ & 3941 & 2724 & 4 & - & 0 & - & 0 \\
\hline $\begin{array}{l}\text { E. coli with genes } \\
\text { of A. tumefaciens }\end{array}$ & 4033 & 2588 & 6 & 5 & 0 & 0 & 1 \\
\hline $\begin{array}{l}\text { E. coli with genes } \\
\text { of } M \text {. tuberculosis }\end{array}$ & 4018 & 2589 & 5 & 4 & 0 & 0 & 1 \\
\hline $\begin{array}{l}\text { E. coli with genes } \\
\text { of S. pyogenes }\end{array}$ & 4015 & 2587 & 6 & 5 & 1 & 0 & 0 \\
\hline
\end{tabular}

(1) After two BLASTN alignments on pangenomes without RNA. (2) After filtering out CDSs of the known GMO databank that are too close to the host species (paragraph 3 of section 2.2). (3) In "potential GM inserts". The "DUGMO final results" column details the results obtained after combining the results of the RF and logit methods, using the data from the learning data and prediction data columns. (4) Estimation of the maximum number of false negatives: the true number cannot be deduced because of the unknown origins of CDSs, potentially from the Bacillales family. (-) Does not apply

$1650 \mathrm{nt}$ or more for those of the pangenome). In the case of incorporation of a highly truncated protein from a protein family poorly represented in the host genome, this sequence may be considered a GM insert. DUGMO makes it possible to target the insert CDSs if the unknown genome submitted is in fact a GM bacterium.

To complete the evaluation of our tool, DUGMO was tested on 6 additional other wild type bacteria: Campylobacter jejuni, Lactococcus lactis, Listeria monocytogenes, Mycobacterium tuberculosis, Salmonella typhimurium and Staphylococcus aureus to check that almost no false positive was present in the results. Then, DUGMO was evaluated on 45 additional tests on synthetic GM samples based on these six bacteria with seven external genes including five genes from these exogenous bacteria, one human gene and one rice gene. Each gene was inserted between two CDS in a reference genome. Then, ART 2.5.8 [36] was used on each modified genome using art_illumina progam with parameters: -ss HS25, -p, $-\mathrm{l} 150,-\mathrm{f} 11,-\mathrm{m} 200,-\mathrm{s} 10$ to create synthetic illumina sequencing data. All the pangenomes used in these tests can be used by users for GM analyses. The pangenomes are provided and the results are detailed in Additional file 1, section 8 .

Among the 6 tests on wild type bacteria, we obtained only one false positive, a $M$. tuberculosis gene that we strongly suspect to come from a recent horizontal gene transfer. Indeed, it does not have megablast [37] alignment with a better identity than $94.7 \%$ in all genomes of actinomycetes found in NCBI wgs database (01/05/2020). Among the 45 synthetic GM tests, we obtained as few as one false positive, probably due to the open S. aureus pangenome and to the environmental and similar genome characteristics of the inserted C. jejuni gene (details in Additional file 1 section 8.6).

Then, we assessed the limits of the method with specific synthetic data. We evaluated the robustness of the method to dicodon optimisation. For this purpose, a B. subtilis gene encoding a riboflavin synthase, optimised for E. coli dicodon usage, was randomly inserted into the reference genome of $E$. coli. Illumina sequencing data were then generated from this genome with ART with the parameters previously described. This 
procedure was run 10 times. In all cases, DUGMO detected the optimised B. subtilis gene as a GM insert, thus proving the method's insensitivity to dicodon optimisation, therefore codon optimisation.

To evaluate the proportion of substitution needed to make a wild type gene detectable by DUGMO, we artificially introduced substitutions in a wild type gene of B. subtilis. Two wild genes were tested independently, a short (417 nucleotide sequence) and a long sequence (1317 nucleotides). The Surfactin long gene and CadI short gene from the wild type $B$. subtilis genome underwent $n$ random substitutions, avoiding mutation events that would be too easily detectable by DUGMO such as substitutions in the start codon, the stop codons,and those that introduce an early stop codon in the sequence. Each modified gene was then replaced into the wild type $B$. subtilis assembly. Finally, Illumina sequencing data were generated using ART [36] software as described above. This process was repeated 10 times. The DUGMO results indicate that beyond $9 \%$ mutations, a mutated CDS is detected as GM.

\section{Discussion}

We propose a method to detect undescribed GM bacteria. DUGMO is intended to assess the presence of bacterial GM following purification of bacteria, as processed in Paracchini et al. [18]. Once a suspected GM is confirmed by DUGMO, PCR targeting the sequence identified by DUGMO can be employed for routine detection of GM in food or environment samples. DUGMO uses both pangenome and genome species properties to feed machine learning and distinguish GM CDSs from host CDSs. The robustness of the pangenome relies heavily on its completeness achievement, and also on accurate genomes attribution to their respective species when they are submitted to public databanks. Species attribution is most often deduced from 16S RNA leading to frequent errors in attribution and then resulting potentially in mixed species pangenomes [38]. The introduction of synthetic strains in the pangenome may also lead to biased statistical properties, due to inconsistent BLAST matches in the host CDSs sorting steps (section 2.3). This highlights the urgent need for tools dedicated to the assessment of the completeness of a pangenome [39].

Recently, Berbers et al. [40] described a revision of an unauthorized GM B. subtilis strain 2014-3557 with one pGMrib plasmid and a $53 \mathrm{~kb}$ chromosomal insertion, firstly described (Data1) by Paracchini et al. [18] with four plasmids pGMBsub01-04. For sake of clarity, in following results, we provide within parentheses gene names used in Paracchini et al.. The GM B. subtilis contains one incorporated plasmid pGMrib, designed for vitamin B2 production (riboflavin) and several chromosomal modifications. First, the cat gene conferring resistance to chloramphenicol is inserted into the chromosomal recA gene. Second, a $53 \mathrm{~kb}$ insertion occurs within the $s c p A$ gene. Within this integration, several occurrences of the full ribDEAHT and partial ribDEA operons show high similarity to the rib operon of Bacillus amyloliquefaciens. In addition, multiple copies of beta-lactamase and kanamycin resistance genes are found in the $53 \mathrm{~kb}$ insertion. Lastly, a bleomycin resistance gene is present.

Both the cat and recA portions of the chromosome are detected as GM CDSs by DUGMO. One B. subtilis gene is discarded by the machine learning step, the chromosomal RNA polymerase delta subunit. DUGMO distinguishes the GM B. amyloliquefaciens $s c p B$ CDS of the chromosomal insert from the wild B. subtilis $s c p B$ of the pGMrib 
plasmid. The pGMrib plasmid (plasmid visualization in Additional file 1, section 9) includes two portions. The first one has a pure B. subtilis ribDEATH operon that is recognized and discarded by DUGMO and includes sipS (lepB) and GAY71_RS22375 (gerPA). The second one carries bacilli genes conferring resistance to erythromycin. In this second portion, nine genes are recognized as GM by DUGMO (GAY71_RS22270 (repS), bin3 / GAY71_RS22275 (beta), soj (delta), zeta, GAY71_RS22265 (copS), GAY71_RS22365 (ORF psi), GAY71_RS22350 (ORF phi), GAY71_RS22355 portion (ORF chi), and GAY71_RS22370 (hyprORF-t)), while the other ten are discarded (GAY71_RS22250 (eta), GAY71_RS22255 (theta), GAY71_RS22260 (iota), topB I GAY71_RS22280 (gamma), GAY71_RS22290 / GAY71_RS22330 (omega), GAY71_ RS22295 / GAY71_RS22325 (epsilon), erm(B), GAY71_RS22315 with additional portion from nucleotides 14,107 to 14,138 (ermC), GAY71_RS22340 (tau-gamma) and GAY71_ RS22345 (ypsilon)). Among them, the ermC and tau-gamma genes are discarded at the BLASTN steps on the pangenome; interestingly, these genes have a nucleotide sequence very close to the CDSs of B. subtilis and are included in host CDSs learning data. Most of the other discarded CDSs match best with enterococci genes, which are gram+ bacilli phylogenetically close to B. subtilis. Part of pGMrib (pGMBsub04 of Paracchini et al.) is thought to derive from the patented pMX45 plasmid for which only B. subtilis origins and genetic engineering methods are mentioned [18]. However, some plasmids have a very wide host range and asserting their origin is not always possible. In this case, the genetic distance of these ten genes, if they were not of B. subtilis origin, does not allow their discrimination. Interestingly, an additional CDS, not described in pGMrib (pGMBsub04 portion) is found twice by DUGMO, once on the plasmid and once on the chromosome. This CDS is present at positions 2652 to 2822 in pGMrib, included in GAY71_RS22250 (1 to 171 in the pGMBsub04 plasmid), and corresponds to a hypothetical protein of an Enterococcus faecalis plasmid (accession number AP018546.1). In addition pGMrib (pGMBsub03 portion) has two additional non B. subtilis genes, part of GAY71_RS22445 (repB) and a shortened version of tet $(L)($ tetR) found GM in DUGMO results. The $53 \mathrm{~kb}$ chromosomal insertion (pGMBsub01 portion) carries genes of different origins (Bacillus amyloliquefaciens and Staphylococcus aureus). The S. aureus genes GAY71_RS12530 (bleR) and aadD1 (kanR) and the ribD, ribA, ribT and $s c p B$ CDSs of the B. amyloliquefaciens ribDEATH operon are correctly detected as GM CDSs by DUGMO. The gene of beta lactamase TEM-116 (ampR / ampiR, six loci in chromosome, one in pGMrib), ribH and scpA CDSs align with the pangenome CDSs in respect to BLASTN parameters, and are included in host CDSs learning data, showing the limits of CDS categorisation for closely related species. This probably also explains the non-detection of rep and ribE. Prokka annotation provides two additional hypothetical CDSs, matching positions 4759-5103 and 10,112-10,201 of the $B$. amyloliquefaciens ribDEATH operon (pGMBsub01 portion) (loci GAY71_ RS12515, GAY71_RS12560, GAY71_RS12605, GAY71_RS12650, GAY71_RS12725, GAY71_RS12770 and GAY71_RS12480, GAY71_RS12690 respectively in the chromosome). The second is labelled GM and the first not.

DUGMO, on the E. coli strain carrying genes of S. pyogenes, found as a false positive CDS that corresponds to a strongly truncated arlS CDS (coding for the arylsulfatase protein), whose full-length version is naturally present in the E. coli genome. During the analysis, the BLASTN on the pangenome CDSs step verify that the lengths of 
matching CDSs do not vary by more than $15 \%$. For the other two E. coli genomes, carrying the genes of $A$. tumefaciens or $M$. tuberculosis, the arlS CDS is full length in the assemblies and is not detected by DUGMO. The truncated arlS CDS in E. coli carrying genes of S. pyogenes is localized at the end of a de novo contig, and the $15 \%$ missing part is found in another contig. Therefore, DUGMO is able to detect CDSs that may consist of a long deletion or insertion in a wild CDS. The mean coverage depth of data for this sample is 41 , while the coverage depth mean of the other two E. coli strains is 63 and 68, respectively. This example indicates that the assembly with an average overlap depth of 41 is partial and leads to a false positive, due to the truncation of a CDS. On the other hand, data assemblies with a coverage depth mean greater than 60 do not have gene truncation problem in our datasets. Most of bacterial assemblers recommend a coverage depth between 80 [41] and 100 (N50 was shown to increase with the coverage depth until a value of 100 for bacterial genomes [42]). A coverage depth of 60 is potentially the acceptable lower limit to obtain a complete assembly without CDS truncation. These observations emphasize the need to obtain a good assembly, and therefore a sufficient coverage depth. In a future version, DUGMO will be able to accept assemblies, including long read assemblies, removing false positive cases due to assembly truncations.

The process of GM bacteria analysis described by Paracchini et al. [18] required more than one month of work (personal communication), while DUGMO needed three hours with 10 threads of a core i7 CPU with 64 GB RAM. The speed of data processing is a major advantage of this tool, which furthermore, does not require prior GMO expertise for end users except to validate the limited set of probable GM genes detected in results. User must have Linux command line skills and have enough biological knowledge to construct a pangenome. In addition, DUGMO has the potential for continuous improvement with the possibility, for the end users, to add new confirmed GMO CDSs to the learning data, and new wild type genomes and CDSs in the pangenome.

\section{Conclusions}

The proposed DUGMO tool combines a high-throughput sequencing data cleaning pipeline with BLASTN alignments on pangenomes, and different Bray-Curtis distance calculations associated with a combination of machine learning methods, the Random Forest and Generalized Linear Model, selected to be the most predictive for GM bacteria data. The tool requires a reliable species-specific pangenome and a reference genome, member of the suspected GM species. Tested on a GM B. subtilis and three GM E. coli, DUGMO is able to detect exogenous or truncated or fused GM CDS and generates few or no false positives and false negatives. This tool is a proof of concept about the detectability of unknown, single, GM bacteria, based on selected properties of the wild type genome. As DUGMO uses statistical properties related to third positions of CDSs, specific to codon usage, it will not detect GM bacterium with only tRNA or rRNA inserts. Conceptually, DUGMO is made to find CDS that do not use the vocabulary of the host genome. DUGMO is not able to distinguish horizontal transferred gene and GM gene. The usage of DUGMO may be deflected to find in wild type bacteria a gene acquired by recent horizontal gene transfer. This DUGMO usage was not tested. We plan to extend 
DUGMO to plant and animal GMO detection by adapting the parameters of distance calculations. Finally, for the first time, DUGMO enables fast, systematic detection of unknown GM bacteria in suspicious samples.

\section{Supplementary information}

Supplementary information accompanies this paper at https://doi.org/10.1186/s12859-020-03611-5.

Additional file 1. Supplementary material.

Additional file 2. Databank of known GMO CDSs.

Additional file 3. List of papers from which known bacterial GM CDSs of the databank were deduced from (format is tabulation separated text file .tsv).

\section{Abbreviations}

GM: Genetically modified; GMO: Genetically modified organism; CDS: Coding sequence; PCR: Polymerase Chain Reaction; BC: Bray-Curtis; RF: Random Forest; logit: Generalized Linear Model

\section{Acknowledgments}

We are grateful to the Joint Research Centre (JRC), the Hessian State Laboratory (LHL) and the Bavarian Health and Food Safety Authority (LGL) for providing us with GM Bacillus subtilis NGS data. The authors thank Yannick Blanchard and Nolwenn Dheilly for their fruitful comments.

\section{Availability and requirements}

Project name: DUGMO (Detection of Unknown Genetically Modified Organisms).

Project home page: http://github.com/ANSES-Ploufragan/DUGMO.git.

Operating system(s): Linux / Unix.

Programming language: Python, $R$.

Other requirements: conda, Python 3.6 or higher. The other dependencies of the program are managed by the conda environment.

License: GNU GPL.

Any restrictions to use by non-academics: no.

\section{Authors' contributions}

$\mathrm{JH}$ designed the method with assistance from FT and developed the DUGMO application. SS and SB helped with the statistical methodology and wrote the statistical part of the paper. MR and MP provided information on GM data and results. JH wrote the biological part and the computational part of the paper with assistance from FT. All results were interpreted by JH. All authors revised and approved the final manuscript.

\section{Funding}

JH was supported by a PhD fellowship from the "région Bretagne" and ANSES. The funding body did not play any roles in the design of the study and collection, analysis, and interpretation of data and in writing the manuscript.

\section{Availability of data and materials}

All data generated or analysed during this study are included in this published article and its supplementary information files.

\section{Ethics approval and consent to participate}

Not applicable.

\section{Consent for publication}

Not applicable.

\section{Competing interests}

The authors declare that they have no competing interests.

\section{Author details}

${ }^{1}$ ANSES, Laboratoire de Ploufragan, GVB unit, 22440 Ploufragan, France. ${ }^{2}$ Université Paris-Saclay, INRAE, MalAGE, 78350 Jouy-en-Josas, France. ${ }^{3}$ ANSES, Laboratoire de Ploufragan, EPISABE unit, 22440 Ploufragan, France. ${ }^{4}$ ANSES, Laboratoire de la santé des végétaux, 49000 Angers, France. ${ }^{5}$ European Commission, Joint Research Centre (JRC), Ispra, Italy.

Received: 11 December 2019 Accepted: 17 June 2020

Published online: 06 July 2020

\section{References}

1. Holst-Jensen A, Bertheau Y, de Loose M, Grohmann L, Hamels S, Hougs L, et al. Detecting un-authorized genetically modified organisms (GMOs) and derived materials. Biotechnol Adv. 2012;30(6):1318-35.

2. EUR-Lex - 32003R1829 - EN - EUR-Lex [Internet]. [cited 2019 Jul 30]. Available from: https://eur-lex.europa.eu/legalcontent/EN/ALL/?uri=CELEX:32003R1829. 
3. Bonfini L, Van den Bulcke MH, Mazzara M, Ben E, Patak A. GMOMETHODS: the European Union database of reference methods for GMO analysis. J AOAC Int. 2012;95(6):1713-9.

4. The European GMO database [Internet]. [cited 2019 Jul 24]. Available from: http://www.euginius.eu/euginius/pages/home.jsf.

5. Petrillo M, Angers-Loustau A, Henriksson P, Bonfini L, Patak A, Kreysa J. JRC GMO-amplicons: a collection of nucleic acid sequences related to genetically modified organisms. Database J Biol Databases Curation. 2015;2015. https://doi.org/10. 1093/database/bav101.

6. Morisset D, Novak PK, Zupanič D, Gruden K, Lavrač N, Žel J. GMOseek: a user friendly tool for optimized GMO testing. BMC Bioinformatics. 2014;15:258.

7. Fraiture M-A, Herman P, Taverniers I, De Loose M, Deforce D, Roosens NH. Current and new approaches in GMO detection: challenges and solutions. Biomed Res Int. 2015;2015:392872.

8. Broeders SRM, De Keersmaecker SCJ, Roosens NHC. How to deal with the upcoming challenges in GMO detection in food and feed. J Biomed Biotechnol. 2012;2012:402418.

9. Fraiture M-A, Herman P, De Loose M, Debode F, Roosens NH. How can we better detect unauthorized GMOs in food and feed chains? Trends Biotechnol. 2017;35(6):508-17.

10. Fraiture M-A, Saltykova A, Hoffman S, Winand R, Deforce D, Vanneste K, et al. Nanopore sequencing technology: a new route for the fast detection of unauthorized GMO. Sci Rep. 2018;8(1):7903.

11. Willems S, Fraiture M-A, Deforce D, De Keersmaecker SCJ, De Loose M, Ruttink T, et al. Statistical framework for detection of genetically modified organisms based on next generation sequencing. Food Chem. 2016;192:788-98.

12. Karlin S, Mrázek J, Campbell AM. Codon usages in different gene classes of the Escherichia coli genome. Mol Microbiol. 1998;29(6):1341-55.

13. Touzain F, Petit M-A, Schbath S, El Karoui M. DNA motifs that sculpt the bacterial chromosome. Nat Rev Microbiol. 2011; 9(1):15-26.

14. Langille MGI, Brinkman FSL. Bioinformatic detection of horizontally transferred DNA in bacterial genomes. F1000 Biol Rep. 2009;: :25.

15. Trifonov V, Rabadan R. Frequency Analysis Techniques for Identification of Viral Genetic Data. mBio. 2010;1(3):e00156-10.

16. Doudna JA, Charpentier E. The new frontier of genome engineering with CRISPR-Cas9. Science. 2014;346(6213):1258096.

17. GM Bacillus subtilis. 2014 [cited 2019 Jul 24]; Available from: http://data.europa.eu/89h/2abb5c2b-3ab6-4ce4-b103-cb1c5 fc7349e.

18. Paracchini V, Petrillo M, Reiting R, Angers-Loustau A, Wahler D, Stolz A, et al. Molecular characterization of an unauthorized genetically modified Bacillus subtilis production strain identified in a vitamin B2 feed additive. Food Chem. 2017;230:681-9.

19. Ames JR, Muthuramalingam M, Murphy T, Najar FZ, Bourne CR. Expression of different ParE toxins results in conserved phenotypes with distinguishable classes of toxicity. MicrobiologyOpen. 2019;8(10):e902.

20. Brito PH, Chevreux B, Serra CR, Schyns G, Henriques AO, Pereira-Leal JB. Genetic competence drives genome diversity in Bacillus subtilis. Genome Biol Evol. 2017;10(1):108-24.

21. Touzain F, Denamur E, Médigue C, Barbe V, El Karoui M, Petit M-A. Small variable segments constitute a major type of diversity of bacterial genomes at the species level. Genome Biol. 2010;11(4):R45.

22. European Commission, Joint Research Centre (2019): GMO-Amplicon sources. European Commission, Joint Research Centre (JRC) [Dataset] PID: http://data.europa.eu/89h/f7e6917f-ccc4-4c88-a622-07c8f961083e.

23. Baron S, Le Devendec L, Touzain F, Jouy E, Lucas P, de Boisséson C, et al. Longitudinal study of Escherichia coli plasmid resistance to extended-spectrum cephalosporins in free-range broilers. Vet Microbiol. 2018;216:20-4.

24. Seemann T. Faster SPAdes assembly of Illumina reads. Contribute to tseemann/shovill development by creating an account on GitHub [Internet]. 2019 [cited 2019 Jul 25]. Available from: https://github.com/tseemann/shovill.

25. Bankevich A, Nurk S, Antipov D, Gurevich AA, Dvorkin M, Kulikov AS, et al. SPAdes: a new genome assembly algorithm and its applications to single-cell sequencing. J Comput. 2012;19(5):455-77.

26. Seemann T. Prokka: rapid prokaryotic genome annotation. Bioinforma Oxf Engl. 2014;30(14):2068-9.

27. Bolger AM, Lohse M, Usadel B. Trimmomatic: a flexible trimmer for Illumina sequence data. Bioinforma Oxf Engl. 2014; 30(15):2114-20.

28. Langmead B, Salzberg SL. Fast gapped-read alignment with bowtie 2. Nat Methods. 2012;9(4):357-9.

29. Li H, Durbin R. Fast and accurate short read alignment with burrows-wheeler transform. Bioinforma Oxf Engl. 2009; 25(14):1754-60.

30. Li H, Handsaker B, Wysoker A, Fennell T, Ruan J, Homer N, et al. The sequence alignment/map format and SAMtools. Bioinforma Oxf Engl. 2009;25(16):2078-9.

31. Altschul SF, Gish W, Miller W, Myers EW, Lipman DJ. Basic local alignment search tool. J Mol Biol. 1990;215(3):403-10.

32. Schbath S, Hoebeke M. R'MES: A Tool to Find Motifs with a Significantly Unexpected Frequency in Biological Sequences. In: Advances in Genomic Sequence Analysis and Pattern Discovery [Internet]. WORLD SCIENTIFIC; 2011 [cited 2019 Jul 23]. p. 25-64. (Science, Engineering, and Biology Informatics; vol. Volume 7). Available from: https://www.worldscientific. com/doi/abs/10.1142/9789814327732_0002.

33. Ricotta C, Podani J. On some properties of the bray-Curtis dissimilarity and their ecological meaning. Ecol Complex. 2017:31:201-5

34. Kuhn M. The caret Package [Internet]. [cited 2019 Jul 23]. Available from: https://topepo.github.io/caret/

35. Baldi P, Brunak S, Chauvin Y, Andersen CAF, Nielsen H. Assessing the accuracy of prediction algorithms for classification: an overview. Bioinformatics. 2000;16(5):412-24.

36. Huang W, Li L, Myers JR, Marth GT. ART: a next-generation sequencing read simulator. Bioinformatics. 2012;28(4):593-4.

37. Madden T. The BLAST Sequence Analysis Tool. 2002 Oct 9 [Updated 2003 Aug 13]. In: McEntyre J, Ostell J, editors. The NCBI Handbook [Internet], National Center for Biotechnology Information (US). Bethesda (MD); 2002. p. Chapter 16. Available from: http://www.ncbi.nlm.nih.gov/books/NBK21097/.

38. Tortoli E. Standard operating procedure for optimal identification of mycobacteria using $16 \mathrm{~S}$ rRNA gene sequences. Stand Genomic Sci. 2010;3(2):145-52.

39. Computational Pan-Genomics Consortium. Computational pan-genomics: status, promises and challenges. Brief Bioinform. 2018;19(1):118-35. 
40. Berbers B, Saltykova A, Garcia-Graells C, Philipp P, Arella F, et al. Combining short and long read sequencing to characterize antimicrobial resistance genes on plasmids applied to an unauthorized genetically modified Bacillus. Sci Rep. 2020;10(1):4310.

41. Chevreux B, Wetter T, Suhai S. Genome sequence assembly using trace signals and additional sequence information. In: Computer science and biology: proceedings of the German conference on bioinformatics (GCB) 99; 1999. p. 45-56.

42. Desai A, Marwah VS, Yadav A, Jha V, Dhaygude K, Bangar U, et al. Identification of Optimum Sequencing Depth Especially for De Novo Genome Assembly of Small Genomes Using Next Generation Sequencing Data. PLOS ONE. 2013; 8(4):e60204.

\section{Publisher's Note}

Springer Nature remains neutral with regard to jurisdictional claims in published maps and institutional affiliations.

Ready to submit your research? Choose BMC and benefit from:

- fast, convenient online submission

- thorough peer review by experienced researchers in your field

- rapid publication on acceptance

- support for research data, including large and complex data types

- gold Open Access which fosters wider collaboration and increased citations

- maximum visibility for your research: over $100 \mathrm{M}$ website views per year

At $\mathrm{BMC}$, research is always in progress.

Learn more biomedcentral.com/submissions 